\title{
High-quality studies needed to determine optimum dental check-up recall intervals
}

\author{
Abstracted from \\ Riley P, Worthington HV, Clarkson JE, Beirne PV.
}

Recall intervals for oral health in primary care patients. Cochrane Database Syst Rev 2013;12: CD004346. doi: 10.1002/14651858.CD004346. pub4. PubMed PMID: 24353242.

Address for correspondence: Cochrane Oral Health Group, School of Dentistry, The University of Manchester, Coupland 3 Building, Oxford Road, Manchester, M13 9PL, UK. E-mail: cohg@manchester.ac.uk

\section{Question: What is the optimum time period to have between one dental check-up and the next?}

Data sources Cochrane Oral Health Group's Trials Register, CENTRAL, Medline via OVID and EMBASE via OVID to 27 Sept 2013; ongoing trials were searched for, reference lists from relevant articles searched and some authors contacted for additional information. There were no restrictions on language or date of publication.

Study selection Randomised controlled trials (RCTS) assessing the effects of different recall intervals.

Data extraction and synthesis Two review authors independently assessed the studies for inclusion, extracted data and carried out risk of bias assessment.

Results Only one study involving 185 participants (adults and children) was included. It compared clinical examination at 12 months with one at 24 months for caries increments ( $\mathrm{dmfs} / \mathrm{DMFS}$ ) and economic cost outcomes (total time used per person).

For caries as an outcome, in three- to five-year olds, the mean difference in dmfs increment was $-0.90(95 \% \mathrm{Cl}-1.96$ to 0.16$)$ and in 16 - to 20-year olds, the mean difference in DMFS increment was -0.86 (95\% $\mathrm{Cl}-1.75$ to 0.03 ) both in favour of a 12-month recall interval.

For time/cost outcomes, in three- to five-year-olds the mean difference in time per participant was ten minutes ( $95 \% \mathrm{Cl}-6.7$ to 26.7 ) and in 16 to 20 -year olds it was 23.7 minutes ( $95 \% \mathrm{Cl} 4.12$ to 43.28 ), again both in favour of 12-month recall interval.

The study was assessed at high risk of bias and contained insufficient evidence to determine whether 12 - or 24 -month recall with clinical examination results in improved caries or time/cost outcomes.

Conclusions There is a very low quality body of evidence from a single RCT. This was insufficient for drawing any conclusions about the potential beneficial and harmful effects of different dental check-up recall intervals. There is no evidence to support or refute practice of six-monthly intervals. High-quality RCTs are needed to address the question of optimum dental check-up recall intervals.

This paper is based on a Cochrane Review published in the Cochrane Library 2013, issue 12 (see www.thecochranelibrary.com for information). Cochrane Reviews are regularly updated as new evidence emerges and in response to feedback, and the Cochrane Library should be consulted for the most recent version of the review.

\section{Commentary}

This systematic review, and its two predecessors published in 2005 and 2007, aimed to establish from existing evidence, the beneficial and harmful effects of different recall intervals for patients seen in primary dental care. This research question has high significance for policy-makers, public health practitioners and clinicians in primary dental care setings around the world. Yet, despite the importance of the topic, this review included only one RCT, which was assessed to be of very low quality.

The included RCT, which was reported in 1992, was conducted in one clinic in Norway with a sample size of 185 patients although no sample size calculation was provided. The participants (children and adults) included in the RCT were deemed to be of low risk of dental diseases and had previously received regular dental care including clinical prevention. These participants were followed up after 24 months based on clinical and cost outcomes with no patient-centred outcomes considered.

The review judged the RCT to be of very low quality due to the lack of blinding and unclear methods of sequence generation and allocation concealment. On a more positive note the systematic review also identified, via trials registers, an ongoing RCT 'INTERVAL' which aims to address the paucity and lack of quality of evidence on this topic. INTERVAL is a multicentre RCT, funded by the National Institute for Health Research, being conducted with dentate adults in the UK.

This trial, based on a sample size calculation, aims to follow 1735 patients for four years and to compare a six-monthly recall interval, risk-based recall and a 24-month recall interval. The outcome measures included are caries, plaque, quality of life, patient satisfaction, anxiety and oral health-related knowledge, attitudes and behaviours, with examiners blinded to the intervention. The RCT is due to end in 2017, which gives hope that when this systematic review is next updated there will be sufficient evidence to make conclusions about the most beneficial interval for dental check-ups in primary dental care.

\section{Practice points}

- There is insufficient evidence to support or refute the need for six-monthly dental check-ups.

Zoe Marshman

School of Clinical Dentistry, University of Sheffield, Sheffield, UK 